\title{
The Effects of Acute Multisession Bifrontal Transcranial Direct Current Stimulation on Intractable Tinnitus and comorbid depression and anxiety: A Study Protocol for Randomized Controlled Trial
}

\author{
Samaneh Rashidi ${ }^{1}$ \\ Ali Yadollahpour ${ }^{1,2 *}$ \\ Saad Ahmed Alkahtani ${ }^{3}$ \\ Pramod Singh kunwar ${ }^{4}$ \\ Daniel Robles-Camarillo ${ }^{5}$ \\ Arturo Meneses-Flores ${ }^{6}$ \\ Francisco Trejo-Macotela ${ }^{5}$
}

\begin{abstract}
Background and Objective: Transcranial Direct Current Stimulation (tDCS) may exert therapeutic effects on tinnitus. This Randomized Control Trial (RCT) with double blind and placebo design investigates the potential therapeutic effects of Transcranial Direct Current Stimulation on chronic and intractable tinnitus using a new protocol. Acute repeated sessions of bifrontal Transcranial Direct Current Stimulation with two months follow up will be used for treatment of tinnitus symptoms and comorbid anxiety and depression. To best of our knowledge, this is the first randomized control trial investigating the effects of acute Transcranial Direct Current Stimulation exposure in intractable tinnitus.

Methods: This randomized control trial is conducted as double blinded placebo controlled with parallel group in patients with chronic intractable tinnitus. The patients will be randomly divided into two real or intervention and sham or placebo Transcranial Direct Current Stimulation. The real Transcranial Direct Current Stimulation group will receive two sessions per day with intersession interval of 6 hours for 5 consecutive days per week for one week (total 10 sessions). The Transcranial Direct Current Stimulation parameters include bifrontal (anode/cathode over right/left dorsolateral prefrontal cortex (DLPFC) $2 \mathrm{~mA}$ current (current density $57.1 \mu \mathrm{A} / \mathrm{cm}^{2}$ ), each session 20 min through $35 \mathrm{~cm}^{2}$ electrodes. The sham group will receive the same Transcranial Direct Current Stimulation parameters and electrode montage and after 30 seconds, the device will be turned off without informing the patient. The primary outcome is the score of Tinnitus Handicap Inventory (THI) measured at baseline (pre-intervention) and post-intervention at immediately, one, and two months after last session. The secondary outcomes are tinnitus loudness, tinnitus distress, depression, and anxiety scores. The tinnitus loudness and distress are measured with a 0-10 Numerical Rating Scale (NRS) preintervention and post-intervention at immediately, one hour, one week, one, and two months after the last Transcranial Direct Current Stimulation session. Beck Depression (BDI-II) and Anxiety Inventories (BAI) is respectively used for measuring depression and anxiety score pre- and post-intervention. Finally, the adverse effects of and tolerability to Transcranial Direct Current Stimulation will be assessed in both real and sham groups using customized questionnaire.

Discussion: To the best of our knowledge this is the first RCT investigating the effects of daily two sessions of Transcranial Direct Current Stimulation in repeated regime on chronic intractable tinnitus symptoms, depression, anxiety, and adverse effects in a long term study of two months follow-up.
\end{abstract}

Keywords: transcranial direct current stimulation, chronic tinnitus, depression, anxiety, acute stimulation.

Abbreviations: ISI: Intersession Interval; tDCS: Transcranial Direct Current Stimulation; DLPFC: Dorsolateral Prefrontal Cortex; THI: Tinnitus Handicap Inventory; BDI-II: Beck Depression Inventory; BAl: Beck Anxiety Inventory; dBHL: Decibels Hearing Level.

\footnotetext{
'Bioelectromagnetic Clinic, Imam Khomeini Hospital, Ahvaz Jundishapur University of Medical Sciences, Ahvaz, Iran

'Department of Medical Physics, School of Medicine, Ahvaz Jundishapur University of Medical Sciences, Ahvaz, Iran

${ }^{3}$ Department of Clinical Pharmacy, College of Pharmacy, Najran University, Kingdom of Saudi Arabia

${ }^{4}$ Department of Pharmaceutics and Pharmacy Practice, School of Pharmacy, Mount Kenya University, Thika, Kenya

${ }^{5}$ Graduate and Research Department, Polytechnic University of Pachuca, Zempoala, Mexico

${ }^{6}$ Information Technologies and Communications Department, Technological University of Huasteca Hidalguense, Huejutla, Mexico

*send correspondence to:

Ali Yadollahpour

Department of Medical Physics and Bioelectromagnetic Clinic-Imam Khomeini Hospital, Ahvaz Jundishapur University of Medical Sciences, Ahvaz, Iran, E-mail:

yadollahpour.a@gmail.com

Paper submitted to the ITJ-EM (Editorial Manager System) on November 22, 2018; and accepted on January $20,2019$.
} 


\section{INTRODUCTION}

Tinnitus is the conscious perception of an auditory phantom sensation without any corresponding external sound source ${ }^{1}$. The perception is usually elementary such as ringing, hissing, and buzzing, but in some cases is of more complex nature such as voices or music. Tinnitus is sometimes be a rhythmical or pulsatile sound ${ }^{2}$. The global prevalence ranges $10 \%-15 \%$ among adults and is usually accompanied by various comorbidities such as depression, anxiety, and sleep disturbances, making it a debilitating condition that can severely affect the quality of life ${ }^{3}$. Neuroimaging and electrophysiological evidence indicate that tinnitus is a heterogeneous disorder characterized by abnormal neural activities in primary and secondary auditory pathways as well as in nonauditory regions such as frontal cortex ${ }^{3,4}$. A mal-adaptive plasticity in the primary and secondary auditory networks may be the main cause of tinnitus. However, other regions particularly frontal cortex is involved in the tinnitus etiology. Frontal cortex, particularly Dorsolateral Prefrontal Cortex (DLPFC) plays important role in attentional and emotional aspects of tinnitus ${ }^{5-7}$.

Despite of different medications administrated for tinnitus, there is no definitive cure for the disorder so that a significant portion of patients do not respond to the medications ${ }^{1,3}$. Therefore, studies are ongoing to develop new medications for tinnitus. However, considering the side effects of the medications, a line of studies are seeking to develop non-medication modalities for tinnitus treatment. In this regard, different modalities such as cognitive behavioral therapy, noise-masking, hearing aids, neuro feedback training, and brain stimulation techniques have been developed ${ }^{8-10}$.

Transcranial Direct Current Stimulation (tDCS) is a noninvasive and safe neuromodulation technique that has been recently introduced as a potential treatment for different neuropsychiatric disorders such as depression ${ }^{11}$, addictions $^{12,13}$, traumatic brain injury ${ }^{14}$, attention deficit hyperactivity disorder ${ }^{15}$, auditory hallucinations ${ }^{16}$, and chronic pain ${ }^{17}$. Moreover, different studies have shown that tDCS could improve different cognitive functions in healthy individuals ${ }^{18}$.

Considering the pathology of tinnitus and the neurophysiological effects of tDCS, we could expect therapeutic effects from tDCS in tinnitus. There are two main hypotheses explaining the therapeutic effects of tDCS in different neuropsychiatric disorders. The first hypothesis says that tDCS exerts disturbance in the ongoing abnormal neural activities responsible for the impaired function or dysfunction. According to this hypothesis, tDCS should target the areas with abnormal activities to alter the impaired function. The second hypothesis claims that tDCS alters the maladaptive plasticity induced by the disorder in specific regions through inducing plastic changes in the target sites. The hyperactivity and maladaptive plasticity in auditory cortex and anomalies in non-auditory regions particularly DLPFC are the main causes of tinnitus ${ }^{2,3,19}$. Therefore, the two main targeting sites in the tDCS studies on studies were $A C$ and DLPFC ${ }^{20-23}$. In line with these hypotheses, several studies have investigated the effects of tDCS in different protocols and electrode montages on tinnitus ${ }^{17,22,23-27}$. Findings of these studies were promising, though controversial.

Different neural pathways or networks are involved in tinnitus in which each network constitutes a clinical aspect of tinnitus. In this regard, we can expect modulating one of these networks would affect one of these aspects 3 ,19,27. The main clinical aspects of tinnitus are loudness, distress, and laterality.

The first line of tDCS studies focused on $A C$ or left temporoparietal area. DLPFC is involved in different aspects of tinnitus considering its role in the regulating the auditory processing, perception, and auditory attention ${ }^{6,7,28,29}$. Therefore, one can expect that modulating the DLPFC would influence the tinnitus loudness perception or tinnitus related distress.

Previous clinical trials have studied the therapeutic outcomes of tDCS over frontal and prefrontal regions in chronic tinnitus ${ }^{20,21}$. However, the main drawback of these studies was that most of them investigated the effects of single tDCS session and the outcomes were assessed for a very short time ranging some hours to few days. Considering the mechanism of action of tDCS and also the mal-adaptive plasticity in tinnitus, repeated sessions of tDCS may have therapeutic value through inducing plastic changes in the impaired regions. Based on the literature search we performed, no RCT has investigated the therapeutic effects of long term protocol of tDCS in repeated sessions on tinnitus. In addition, most of the studies conducted so far, used a single-sessionper-day protocol. Therefore, this RCT aims to study the treatment effectiveness of twice daily (intersession interval: 6 hours) bifrontal tDCS in repeated sessions on tinnitus symptoms, its loudness and distress as well as on comorbid depression and anxiety in a two-month followup. We hypothesized that acute repeated sessions would result in aggregative therapeutic effects on tinnitus. Also, we expect that bifrontal tDCS will reduce the depression and anxiety scores in the patients. We will evaluate the interactions of tDCS factors and disease characteristics including gender, THI basal score, laterality, tinnitus quality, and duration with the response to the treatment.

\section{METHODS}

\section{Subjects}

This study is a section of a big project we designed in our clinic to comprehensively investigates the therapeutic value of tDCS in tinnitus. During this project we will study the efficacy of tDCS in different protocols applied 
in different brain sites for the treatment of tinnitus. The studies will be conducted in the Bioelectromagnetic Clinic in Ahvaz Imam Hospital, Ahvaz, Iran. The patients with chronic tinnitus ( $>2$ years) who were resistant to the medications are enrolled in this clinical trial. This is a double blind RCT with parallel allocation of two groups.

\section{Ethics approval and study registration}

The procedures of this clinical trial were approved by the ethics committee in the human studies of Ahvaz Jundishapur University of Medical Sciences (AJUMS), Ahvaz, Iran (Ethic code: IR.AJUMS.REC.1394.639). The regulations of the ethics committee were completely coincide with regulations of human studies set by the Helsinki declaration ${ }^{30}$. The researchers will clearly explain the main objectives, experiments, and the assessments of the study and also the possible benefits and risks of participation to all subjects before the intervention and then the written consent form will be collected from all participants. This RCT was registered in the Iranian registry of clinical trials (IRCT2016110124635N5).

\section{Inclusion and exclusion criteria}

After the enrolment of the subjects, the inclusion and exclusion criteria will be evaluated through predefined neurological and audiological examinations by expert specialists. The patients are selected from the tinnitus patients referred to the Tinnitus Clinic in the Khuzestan Cochlear Implant Center, Ahvaz, Iran. The participants have the right to terminate the participation anytime during the study. Moreover, researchers will terminate the procedures for any patients who would report significant Adverse Effects (AEs). The inclusion criteria include idiopathic chronic (with more than 2 years) and medication-resistant tinnitus, age range of 18 to 65 years old, no concurrent administration of medication. The exclusion criteria include history of epileptic seizures, brain trauma, psychotic, severe psychiatric disorders, severe vertigo, Meniere's disease, severe organic comorbidity, using pacemaker or defibrillator, present pregnancy, neurologic disorders such as brain tumors, concurrent treatment administered for mental disorders, and receiving previous treatments with rTMS or tDCS within 6 months. The audiometric and neurologic examinations are performed in the Khuzestan Cochlear Center, Ahvaz, Iran. The intervention and outcome assessments and follow up evaluations will be conducted in the Bio electromagnetic Clinic in Ahvaz Imam Hospital, Ahvaz, Iran.

\section{Study design and intervention}

This is a double-blind RCT in which the patients are randomly assigned into two groups of real tDCS and sham tDCS. To reduce the inter-individual variability and considering the parallel allocation of the two groups, the real and sham groups will be matched for age, gender, ethnicity, and the main audiometric characteristics. In addition, to reduce the subjective bias, the study will be double blinded in which the patients and the researchers who collect and analyze the data are blinded on the type of the intervention. Furthermore, the both real and sham tDCS groups will be assessed for the blinding quality following the last tDCS session. The random allocation is performed using computer software generating random numbers with blocking approach. The allocation concealment is secured using sequentially numbered, opaque, sealed envelopes.

\section{tDCS intervention}

For tDCS session, we will use a battery-driven constant current stimulator (OASIS ProTM device by Mind Alive Inc., Edmonton, Alberta, Canada) that delivers electric direct current up to $2 \mathrm{~mA}$ using a pair of saline-soaked surface electrodes $\left(35 \mathrm{~cm}^{2}\right)$. The exposure protocol in the real tDCS consists of two sessions per day, each session 20 min with inter-stimulation interval (ISI) of 6 hours for five consecutive days ( 10 sessions in total). The current is $2 \mathrm{~mA}$ and the anode/cathode will be centered right (F4)/ left (F3) DLPFC according to the international 10-20 EEG placement system. The sham tDCS will undergo the same electrode montage and the same session parameters of that the real tDCS, except that the tDCS device will be turned off after 30 seconds without the knowledge of the patients to blind them on the type of the tDCS intervention. The rationale for choosing the 30 seconds at the beginning of the session as the fade out period is that previous studies have demonstrated that the AEs and sensations due to tDCS such as skin burning, itching, and tingling disappear after 30 seconds of the active tDCS ${ }^{31,32}$.

\section{ADVERSE EFFECTS}

The dose-response and safety studies have shown that the tDCS protocols with 1-2 mA current and duration of 15-20 min in single session to few sessions result in no serious $\mathrm{AEs}^{31,33}$. The common reported $\mathrm{AEs}$ are itching, pinching, tingling, fatigue, headache, and local burn under the electrodes. In overall, the AEs of tDCS were mild and the technique has been reported as a safe modality. However, this should be noted that most of the studies that assessed the AEs of tolerability to tDCS have been conducted on single tDCS session of 20-30 min duration and 1 to $2 \mathrm{~mA}$ current and electrode size of $35 \mathrm{~cm}^{2}$. In this line, few studies have assessed the safety and tolerability profiles of few sessions (three to five sessions) that showed the same level AEs as of the single session protocols. The patients will undergo repeated sessions of tDCS (10 sessions) that may lead to more severe AEs than the previous studies with single to five sessions. Therefore, we will evaluate the AEs of and tolerability to the tDCS intervention using a researcher built questionnaire (Supplementary Table 1). AEs and tolerability of all patients will be assessed after each tDCS session in both real and sham tDCS (Table 1)

\section{RESULTS}


Table 1. Setting for real and sham tDCS [Note: *30 seconds after the start of the stimulation the device will be turned off without informing the patient].

\begin{tabular}{ccc}
\hline Variables & Real tDCS & Sham tDCS \\
\hline Current density & $57.1 \mu \mathrm{A} / \mathrm{cm}^{2}$ & $57.1 \mu \mathrm{A} / \mathrm{cm}^{2}$ \\
Electrode & Anode right (F4), & Anode right (F4), \\
montage & Cathode left (F3) DLPFS & Cathode left (F3) DLPFS \\
Duration & $20 \mathrm{~min}$ & $30 \mathrm{~S}^{*}$ \\
\hline
\end{tabular}

The data of this clinical trial comprise of three main groups: (1) patients' specific data, (2) response variables, and (3) AEs and tolerability. The patients' specific data are assessed and recorded at the baseline prior to the intervention. The response variables include primary and secondary outcomes and are assessed at baseline and at different time points after tDCS intervention. The AEs and tolerability data are collected after each tDCS session. The SPIRIT schematic protocol of this trial including the patients' specific data and the response variables along with the time course of the assessments for each outcome is represented in Table 2 . The data are measured and recorded at two main time points of preand post-intervention. The pre-intervention assessments are performed before the first tDCS session on the first day of intervention (TO). The post-intervention assessments are performed at five time points including immediately (T1), one hour (T2) one week (T3), one month (T4), and two months (T5) after the last tDCS session (Table 2).

\section{Patients' specific data}

After the enrolment, the patients will undergo complete neurological and audiometric assessments by expert specialties. The hearing threshold and hearing loss type, tinnitus duration, quality, and laterality as well as the age and gender of all patients will be recorded. Tinnitus quality is categorized in different classes including buzzing, cicadas and high pitch whistling, hissing, humming, ringing, pulsating, thumping, and ticking. Audiometric assessments are performed inside an acoustically isolated room (ISO 8253-1:2010). Pure-tone Audiometry (PTA), conducted by an AC 40 dual channel audiometer (Intracoustics Co., Denmark) is used to determine the hearing threshold. The modified HughsonWestlake method recommended by ANSI 1997 is used to determine hearing thresholds over $250-8000 \mathrm{~Hz}$ and 500 $4000 \mathrm{~Hz}$ frequency ranges respectively for air conduction and bone conduction pathways ${ }^{34}$. The hearing class is defined as the averaged threshold at $250 \mathrm{~Hz}, 1000,2000$, and $4000 \mathrm{~Hz}$. The PTA outcome or hearing threshold is normal when the hearing threshold of all frequencies is below $20 \mathrm{dBHL}$. The class of hearing loss for both ears of all patients is determined according to the type and degree follows: normal (<20 dB), mild (20-40 dB), moderate $(41-70 \mathrm{~dB})$, severe $(70-90 \mathrm{~dB})$, and profound hearing loss $(>90 \mathrm{~dB})^{35}$.

\section{Response variables}

The response variables comprised of primary and secondary outcomes as well as the data of the AEs and tolerability Tinnitus Handicap Inventory (THI) score is defined as the primary outcome. The THI is widely used as the primary outcome measure in double-blind RCT to study the efficacy of medications or non-medication modalities in tinnitus patients ${ }^{36}$. In this $\mathrm{RCT}$, we will measure the THI scores at pre-intervention (T0) and at immediately (T1), one month (T4), and two months (T5) after the last tDCS session. For the primary outcome (THI score) we will use the clinical improvement rather than statistical improvement so that a reduction $\geq 20$ points in the THI score (Pre-treatment THI-Post-treatment THI $\geq$ 20 ) is defined as the treatment response. The 20 point cut-off value of THI is generally accepted as significant

Table 2. Standard Protocol Items: Recommendations for interventional trials of this study [Note: THI: Tinnitus Handicap Inventory, BDI: Beck Depression Inventory, BAl: Beck Anxiety Inventory, T0: Before Intervention, T1: Immediately After Last Session, T2: One Hour After Last Session, T3: One Week T4: One Month, and T5: two months after last tDCS session].

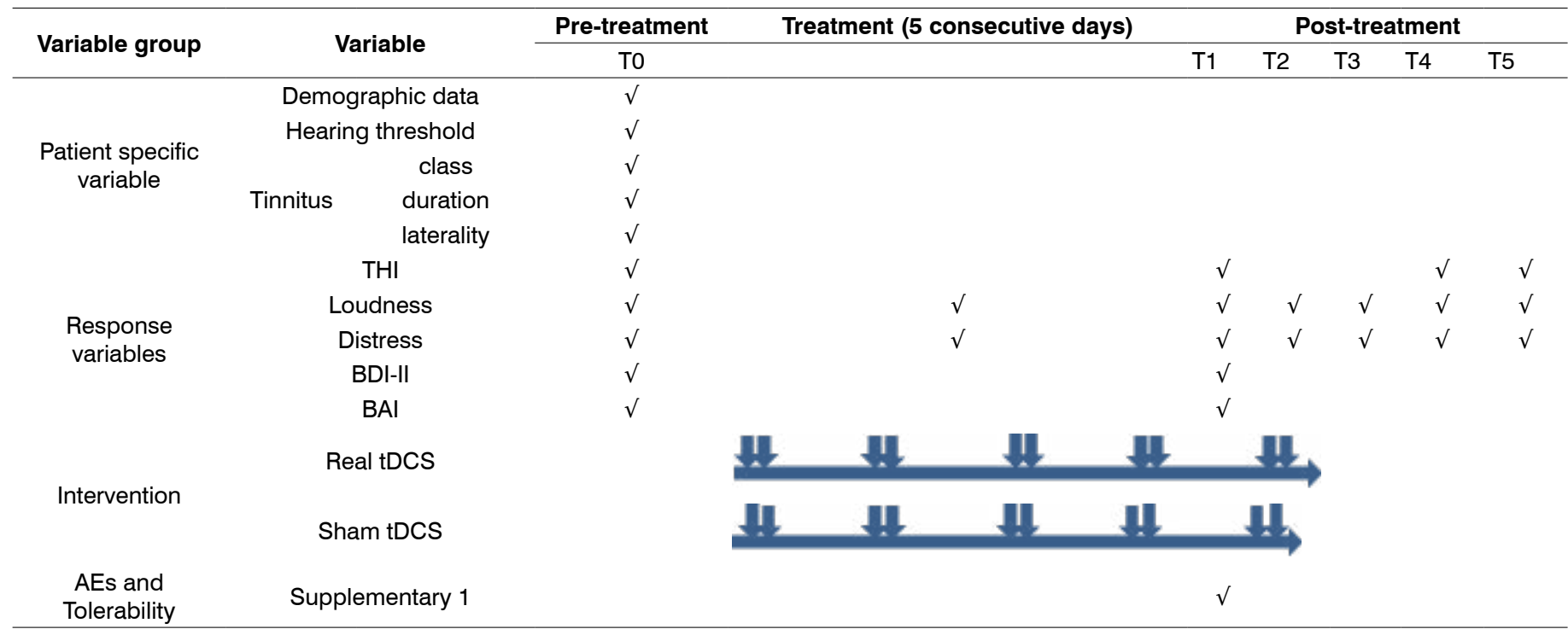


clinical improvement in tinnitus treatment ${ }^{26,37,38}$.

In addition to the primary outcome, four secondary outcomes are defined for this study including tinnitus loudness (intensity), tinnitus related distress, depression, and anxiety. The tinnitus loudness and distress are assessed with a 0-10 NRS at pre-intervention (TO) and at five time points of post-intervention including immediately (T1), one hour (T2) one week (T3), one month (T4), and two months (T5) after the last tDCS session (Table 2). A reduction of 10 percent in the baseline score of loudness and distress, 1 point reduction in the 0-10 NRS, is considered as treatment response ${ }^{39,40}$. The depression and anxiety comorbid with tinnitus are assessed respectively with Beck Depression Inventory-II (BDI-II) and Beck Anxiety Inventories (BAI) at pre- (TO) and immediately post intervention (T1). The treatment response for the BAI and $\mathrm{BDI}$ scores is defined the reduction of equal or higher than $50 \%$ of the baseline (pre-treatment) score ${ }^{40,41}$.

The AEs of and tolerability to the tDCS sessions will be assessed using a customized questionnaire (Supplementary Table 1) after each tDCS session.

\section{Blinding assessment}

For defining the blinding condition for the patients in the sham group, the tDCS device will be turned on for the initial 30 seconds of tDCS session and then without the knowledge of the patient it becomes turned off. This period is based on the previous findings that demonstrated that the perceived tDCS induced sensations usually persist 30 seconds and after that fade out. To assess the quality of the blinding, we will ask all patients in both groups to determine which type of stimulation, real or sham tDCS, they have received. This assessment is conducted along with the AEs after the last tDCS session.

\section{Statistical assessments}

All statistical tests are conducted with Statistical Package for the Social Sciences (SPSS) (version 20, IBM Corporation, New York, USA). Independent samples t-tests are used to compare the differences of age, sex, tinnitus quality tinnitus laterality, THI score, duration, and severity of hearing loss as the dependent variables between the real and sham tDCS groups to evaluate the level of match between the two groups.

For nominal variables such as gender and tinnitus class, the two groups will be compared using Chi-square test. Independent sample t-tests are used to assess whether individual baseline variables including THI, BDI, BAI, and tinnitus loudness and distress differ between the two groups. Levene's test will be used before t-tests to evaluate the equality of variances So that if the Levene's test is not violated, then equality of variances is assumed. The number of female and male participants and the tinnitus quality, across each group will be compared using Pearson Chi-square tests. Mixed repeated-measure ANOVAs with the within-subject factor Time and the between-subject factor Stimulation are used to assess the changes in the primary and secondary outcomes including THI, tinnitus loudness, tinnitus distress, BDI, and BAI scores. For the ANOVAs, sphericity will be tested using the Mauchly's test and if it is violated, then the Greenhouse-Geisser correction is performed. In case of significant effects, follow-up post-hoc t-tests will be conducted with LSD adjustments for multiple comparisons to examine if tDCS caused a significant difference relative to sham or baseline. In addition, the Pearson's correlation coefficients will be calculated between the pre- to postintervention changes of THI and BDI values.

The number of responders versus non-responders for both real and sham groups will be evaluated through calculating pre- to post-intervention changes of THI scores (Vanneste et al. 2010; Vanneste et al. 2011). Responders are those patients who show $\geq 20$ points in THI score (Pre-treatment THI-Post-treatment THI $\geq 20$ ). To determine the differences between the proportions of responders versus non-responders under each condition, we will use the Pearson's chi-square tests. For all statistical analyses, the statistically significance level is set at $p$-value $=0.05$.

\section{Sample size calculation}

We calculated the sample size of this clinical trial with the standard sample size formula (Eq.1). Considering the study of Garin et al. ${ }^{42}$ as the base study, power of $85 \%$ and confidence interval of 95 percent, we calculated the sample size as 25 . To count for any attrition due to long run of the study, we will enroll 30 patients in each group.

$$
n=\frac{\left(Z_{1-\frac{a}{2}}+Z_{1-\hat{a}}\right)^{2}\left(S_{1}^{2}+S_{2}^{2}\right)}{\left(\bar{X}_{1}-\bar{X}_{2}\right)^{2}}
$$

\section{DISCUSSION}

This clinical trial is designed to investigate the treatment effects of multi-session tDCS (anodal/cathodal over the right/left DLPFC) on tinnitus symptoms and comorbid depression and anxiety in patients with medication resistant tinnitus. The tDCS has shown beneficial effects in improving cognitive functions in healthy ${ }^{18}$ and cognitive impaired individuals ${ }^{11,16,43-46}$. Moreover, it has shown beneficial effects for different neuropsychiatric disorders such as depression ${ }^{11}$, obsessive compulsive disorder ${ }^{46}$, auditory-verbal hallucinations ${ }^{16}$, and chronic pain ${ }^{17}$. Several studies have reported potential treatment efficacies of tDCS for chronic tinnitus ${ }^{20,23,24,26,47}$. However, the findings are inconsistent and majority of them have focused on single session protocol investigating transient and short term effects. The main advantages of this study are its double blinded design and the relatively long term follow up period of two months. Moreover, it will concurrently evaluate the effects of the tDCS on the comorbid depression and anxiety in the patients. To the best of our knowledge this will be the first study of 
multisession tDCS with two months follow-up.

\section{DECLARATIONS}

\section{Ethics approval and consent to participate}

The study was approved by the local ethics committee of AJUMS, Ahvaz, Iran (registration code: IR.AJUMS. REC.1394.639). After enrolment and before the start of the study, researcher will clearly explain the experimental procedures, the objectives, possible benefits, and side effects of the study to the patients and then all participants will fill and sign a written consent form for participation in the study.

\section{Trial registration}

The present study was registered at Iranian Registry of Clinical Trials (http://www.irct.ir:IRCT2016110124635N5).

\section{Availability of data and material}

The datasets generated and/or analyzed during the current study are available from the corresponding author on reasonable request. Furthermore, the authors will publish the dataset of this study in the appropriate journal following the completion of this study.

\section{Competing interests}

The authors declare that they have no competing interests.

\section{Acknowledgements}

The authors would like to thank the staff of the Khuzestan cochlear center, Ahvaz, Iran for their assistance in performing the audiometric assessments.

\section{REFERENCES}

1. Sanchez L. The epidemiology of tinnitus. Audiol Med. 2004;2:8-17.

2. Eggermont JJ. Pathophysiology of tinnitus. Prog Brain Res. 2007; 166:19-543.

3. Vanneste S, Plazier M, Van Der Loo E, Van de Heyning P, Congedo $\mathrm{M}$, De Ridder D. The neural correlates of tinnitus-related distress. Neuroimage. 2010;52:470-80.

4. Eggermont JJ. The neuroscience of tinnitus. Oxford University Press; 2012.

5. Bodner M, Kroger J, Fuster JM. Auditory memory cells in dorsolateral prefrontal cortex. Neuroreport. 1996;7:1905-8.

6. Herrington JD, Mohanty A, Koven NS, Fisher JE, Stewart JL, Banich MT, et al. Emotion-modulated performance and activity in left dorsolateral prefrontal cortex. Emotion. 2005;5:200-7.

7. Knight RT, Scabini D, Woods DL. Prefrontal cortex gating of auditory transmission in humans. Brain Res. 1989;504:338-42.

8. Khoramzadeh S, Saki N, Davoodi I, Nosratabadi M, Yadollahpour A. Investigating the therapeutic efficacy of neurofeedback treatment on the severity of symptoms and quality of life in patients with tinnitus. Int J Ment Health Addict. 2016;14:982-92.

9. Langguth B, Kreuzer PM, Kleinjung T, De Ridder D. Tinnitus: causes and clinical management. Lancet Neurol. 2013;12:920-30.

10. Hobson J, Chisholm E, El Refaie A. Sound therapy (masking) in the management of tinnitus in adults. Cochrane Libr. 2010.

11. Yadollahpour A, Jalilifar M, Rashidi S. Transcranial direct current stimulation for the treatment of depression: a comprehensive review of the recent advances. Int J Ment Health Addict. 2017;15:434-43.

12. Yadollahpour A, Tifei Y. Transcranial direct current stimulation for the treatment of addictions: A systematic review of clinical trials. Curr Psychiatry Rev. 2018;14:1.

13. Palacios EM, Lopes IBC, Souza RA, Klauss J, Batista EK, Conti $\mathrm{CL}$, et al. Ventral medial prefrontal cortex (vmPFC) as a target of the dorsolateral prefrontal modulation by transcranial direct current stimulation (tDCS) in drug addiction. J Neural Transm. 2016;123:1179-94.

14. Yoon KJ, Lee YT, Chae SW, Park CR, Kim DY. Effects of anodal transcranial direct current stimulation (tDCS) on behavioral and spatial memory during the early stage of traumatic brain injury in the rats. J Neurol Sci. 2016;362:314-20.

15. Cosmo C, Baptista AF, De Araujo AN, Do Rosario RS, Miranda JG V, Montoya P, et al. A Randomized, Double-Blind, Sham-Controlled Trial of Transcranial Direct Current Stimulation in Attention-Deficit/ Hyperactivity Disorder. PLoS One. 2015;10.

16. Shiozawa P, Da Silva ME, Cordeiro Q, Fregni F, Brunoni AR. Transcranial direct current stimulation (tDCS) for the treatment of persistent visual and auditory hallucinations in schizophrenia: a case study. Brain Stimul. 2013;6:831.

17. Kuo MF, Paulus W, Nitsche MA. Therapeutic effects of non-invasive brain stimulation with direct currents (tDCS) in neuropsychiatric diseases. Neuroimage. 2014;85Pt 3:948-60.

18. Yadollahpour A, Asl HM, Rashidi S. Transcranial direct current stimulation as a non-medication modality for attention enhancement: A review of the literature. Res J Pharm Technol. 2017;10:311-6.

19. Elgoyhen AB, Langguth B, De Ridder D, Vanneste S. Tinnitus: perspectives from human neuroimaging. Nat Rev Neurosci. 2015;16:632-42.

20. Frank E, Schecklmann M, Landgrebe M, Burger J, Kreuzer P, Poeppl TB, et al. Treatment of chronic tinnitus with repeated sessions of prefrontal transcranial direct current stimulation: outcomes from an open-label pilot study. J Neurol. 2012;259:327-33. 21. Vanneste S, Focquaert F, Van de Heyning P, De Ridder D. Different resting state brain activity and functional connectivity in patients who respond and not respond to bifrontal tDCS for tinnitus suppression. Exp Brain Res. 2011;210:217-27.

21. Shekhawat GS, Stinear CM, Searchfield GD. Modulation of perception or emotion? A scoping review of tinnitus neuromodulation using transcranial direct current stimulation. Neurorehabil Neural Repair. 2015;29:837-46.

22. Vanneste S, De Ridder D. Bifrontal transcranial direct current stimulation modulates tinnitus intensity and tinnitus-distress-related brain activity. Eur J Neurosci. 2011;34:605-14.

23. Hyvarinen P, Makitie A, Aarnisalo AA. Self-administered domiciliary tdcs treatment for tinnitus: a double-blind sham-controlled study. PLoS One. 2016;11:e0154286.

24. Joos K, De Ridder D, Van de Heyning P, Vanneste S. Polarity specific suppression effects of transcranial direct current stimulation for tinnitus. Neural Plast. 2014;2014:1-8.

25. Shekhawat GS, Sundram F, Bikson M, Truong D, De Ridder D, Stinear CM, et al. Intensity, duration, and location of high-definition transcranial direct current stimulation for tinnitus relief. Neurorehabil Neural Repair. 2016;30:349-59.

26. Forogh B, Mirshaki Z, Raissi GR, Shirazi A, Mansoori K, Ahadi T. Repeated sessions of transcranial direct current stimulation for treatment of chronic subjective tinnitus: a pilot randomized controlled trial. Neurol Sci. 2016;37:253-9.

27. De Ridder D, Fransen H, Francois O, Sunaert S, Kovacs S, Van De Heyning P. Amygdalohippocampal involvement in tinnitus and auditory memory. Acta Otolaryngol. 2006;126:50-3. 
28. Yuan T, Yadollahpour A, Salgado-Ramírez J, Robles-Camarillo D, Ortega-Palacios R. Transcranial direct current stimulation for the treatment of tinnitus: a review of clinical trials and mechanisms of action. BMC Neurosci. 2018;19:66.

29. Vanneste S, Van de Heyning P, De Ridder D. The neural network of phantom sound changes over time: a comparison between recent onset and chronic tinnitus patients. Eur J Neurosci. 2011;34:718-31.

30. General assembly of the world medical association. World medical association declaration of Helsinki: Ethical principles for medical research involving human subjects. J Am Coll Dent. 2014;81:14-8.

31. Poreisz C, Boros K, Antal A, Paulus W. Safety aspects of transcranial direct current stimulation concerning healthy subjects and patients. Brain Res Bull. 2007;72:208-14.

32. Paulus W. Transcranial direct current stimulation (tDCS). Suppl Clin Neurophysiol. 2003;56:249-54.

33. Boggio PS, Rigonatti SP, Ribeiro RB, Myczkowski ML, Nitsche MA, Pascual-Leone A, et al. A randomized, double-blind clinical trial on the efficacy of cortical direct current stimulation for the treatment of major depression. Int J Neuropsychopharmacol. 2008;11:249-54.

34. Lloyd LL, Kaplan H. Audiometric interpretation: A manual of basic audiometry. University Park Press;1978.

35. Association AS-L-H. Guidelines for manual pure-tone threshold audiometry. 2005.

36. Newman CW, Jacobson GP, Spitzer JB. Development of the tinnitus handicap inventory. Arch Otolaryngol Neck Surg. 1996;122:143-8.

37. Shekhawat GS, Stinear CM, Searchfield GD. Transcranial direct current stimulation intensity and duration effects on tinnitus suppression. Neurorehabil Neural Repair. 2013;27:164-72.

38. Vanneste S, Plazier M, Ost J, Van der Loo E, Van de Heyning $P$, De Ridder D. Bilateral dorsolateral prefrontal cortex modulation for tinnitus by transcranial direct current stimulation: a preliminary clinical study. Exp brain Res. 2010;202:779-85.
39. Vanneste S, Van de Heyning P, De Ridder D. Contralateral parahippocampal gamma-band activity determines noise-like tinnitus laterality: a region of interest analysis. Neuroscience. 2011;199:481-90.

40. Smarr KL, Keefer AL. Measures of depression and depressive symptoms: Beck Depression Inventory, Center for Epidemiologic Studies Depression Scale, Geriatric Depression Scale, Hospital Anxiety and Depression Scale, and Patient Health Questionna. Arthritis Care Res. 2011;63.

41. Julian LJ. Measures of anxiety: State. trait anxiety inventory (STAI), beck anxiety inventory (BAl), and hospital anxiety and depression scale-anxiety (HADS-A). Arthritis Care Res. 2011;63.

42. Garin P, Gilain C, Van Damme JP, De Fays K, Jamart J, Ossemann $M$, et al. Short and long-lasting tinnitus relief induced by transcranial direct current stimulation. J Neurol. 2011;258:1940-8.

43. Smith RC, Boules S, Mattiuz S, Youssef M, Tobe RH, Sershen H, et al. Effects of transcranial direct current stimulation (tDCS) on cognition, symptoms, and smoking in schizophrenia: A randomized controlled study. Schizophr Res. 2015;168:260-6.

44. Brunelin J, Mondino M, Gassab L, Haesebaert F, Gaha L, SuaudChagny MF, et al. Examining transcranial direct-current stimulation (tDCS) as a treatment for hallucinations in schizophrenia. Am J Psychiatry. 2012;169:719-24.

45. Mondino M, Haesebaert F, Poulet E, Saoud M, Brunelin J. Efficacy of cathodal transcranial direct current stimulation over the left orbitofrontal cortex in a patient with treatment-resistant obsessivecompulsive disorder. J ECT. 2015;31:271-2.

46. Yadollahpour A, Bayat A, Rashidi S, Saki N, Karimi M. Dataset of acute repeated sessions of bifrontal transcranial direct current stimulation for treatment of intractable tinnitus: A randomized controlled trial. Data Br. 2017;15:40-6.

47. Shekhawat GS, Stinear CM, Searchfield GD. Transcranial direct current stimulation intensity and duration effects on tinnitus suppression. Neurorehabil Neural Repair. 2013;27:164-72. 